\title{
Multiple Dielectric Relaxations in Solid Polyorganophosphazenes
}

\author{
Shunsuke UzAKI ${ }^{\dagger}$ Keiichiro ADACHI, and Tadao KoTAKA \\ Department of Macromolecular Science, Faculty of Science, \\ Osaka University, Toyonaka, Osaka 560, Japan
}

(Received August 4, 1987)

\begin{abstract}
Dielectric properties of solid films of poly(dichloro phosphazene) (PCPN), poly(diphenoxy phosphazene) (PPPN), and poly(difluoroethoxy phosphazene) (PFPN) were studied in the frequency range from 0.1 to $100 \mathrm{kHz}$. PCPN exhibited only one relaxation peak (named as $\alpha$ process), while PPPN and PFPN exhibited two loss maxima ( $\alpha$ and $\beta$ ) in the dielectric loss $\varepsilon^{\prime \prime}$ curve within the temperature range between 90 and $460 \mathrm{~K}$. The $\alpha$ process for the each sample was seen just above its glass transition temperature $T_{\mathrm{g}}$, while the $\beta$ relaxation was observed only for PPPN and PFPN in the region far below their $T_{\mathrm{g}}$. This result indicates that the $\beta$ process is due to rotation of the side groups. Based on the simplest two site model, we estimated the amplitude of the rotational oscillation of the side groups and the energy difference between the two sites. Besides these $\alpha$ and $\beta$ relaxations, PPPN and PFPN showed the mesophase transition at 400 and $345 \mathrm{~K}$, respectively, where the dielectric constant increased step-wise. The dielectric constant in the mesophase was partly attributable to the polarization of the type-A dipoles.
\end{abstract}

KEY WORDS Polyphosphazene / Poly(organophosphazene) / Poly(dichloro phosphazene) / Poly(diphenoxy phosphazene) / Poly(difluoroethoxy phosphazene) / Dielectric Relaxation / Primary Process / Side Group / Mesophase / Two-Site Model /

Amorphous polymers exhibit multiple dielectric relaxations since they possess various degrees of freedom for the molecular motions. ${ }^{1}$ The processes are usually called $\alpha, \beta, \gamma$, and so on from the one appearing at the highest temperature to those at lower temperatures. We expect that polyorganophosphazene has three types of dipoles being classified by Stockmayer ${ }^{2}$ as types A, B, and C. The type-A dipoles correspond to those aligned in the same direction parallel to the chain contour; the type- $\mathrm{B}$, those aligned in the perpendicular direction; and the type-C, those mobile in the side groups.

In our previous study for dilute solutions of poly(diphenoxy phosphazene) (PPPN), ${ }^{3}$ we found that PPPN exhibits the dielectric re- laxation conforming to the Zimm theory. This indicates that polyphosphazene has the type-A dipoles whose dipole moment was estimated to be $6.7 \mathrm{D}$ per monomer unit from the relaxation strength. However, in dilute solutions, motions of the type- $\mathrm{B}$ and $\mathrm{C}$ dipoles could not be observed since the relaxation times were much shorter than the time scale of our experiment. To study the relaxations due to the types $\mathrm{B}$ and $\mathrm{C}$ dipoles, we have to use either concentrated solutions or bulk films.

At present, a variety of poly(organo phosphazene)s have been synthesized. ${ }^{5-7}$ In this study, we carried out dielectric measurement on bulk films of poly(dichloro phosphazene) (PCPN), PPPN, and poly(difluoroethoxy phosphazene (PFPN). It is obvious

\footnotetext{
${ }^{\dagger}$ Present address: Mitsubishi Electric Co., 8-1-1 Tsukaguchi-Honmachi, Amagasaki 661, Japan.
} 
that PCPN does not possess Type-C dipoles. Therefore, from comparison of the dielectric behavior of PCPN with the other two, we may assign the relaxations due to Type $C$ dipoles in PPPN and PFPN.

Allen et $a l^{8}$ reported that PFPN, poly $(p$ phenylphenoxy phosphazene) and poly( $p$ chlorophenoxy phosphazene) exhibited two dielectric loss maxima around 250 and $120 \mathrm{~K}$. However, they did not analyse the data in detail and only speculated that the low temperature process was due to Type-C relaxation.

One of the characteristic features of polyphosphazene is that although this polymer has properties of a typical flexible chain in dilute solution, ${ }^{3.9}$ it exhibits a mesophasestructure similar to nematic liquid crystals in concentrated solution ${ }^{10}$ and in bulk. ${ }^{5-7,9}$ In this study, the mobility of the polyphosphazene chains in the mesophase was also analyzed.

\section{EXPERIMENTAL}

\section{Samples}

PCPN was prepared by thermal ring opening polymerization of cyclo(hexachloro triphosphazene) supplied from Shin-Nisso Chemicals Co., Ltd. The monomer was sealed in a glass ampule together with $2.5 \%$ of boron trichloride $\left(\mathrm{BCl}_{3}\right)$ in vacuum and allowed to polymerize at 170 to $180 \mathrm{~K}$ for $c a .100 \mathrm{~h}$. PPPN and PFPN were prepared by substitution of chlorine $(\mathrm{Cl})$ atoms of PCPN with organogroups according to the method reported by Allcock and Kugel. ${ }^{5}$ The elemental analysis of PPPN indicated that substitution reaction was almost perfect and the remaining $\mathrm{Cl}$ atoms were only $1.3 \mathrm{~mol}^{\circ} \%$. The elemental analysis of $\mathrm{Cl}$ for PFPN was not successful due to fluorine atoms. However, we estimated the $\mathrm{Cl}$ content to be $c a .1 \%$ from the carbon content. The weight average molecular weight $\bar{M}_{w}$ and the ratio of $\bar{M}_{w}$ to the number average molecular weight $\bar{M}_{n}$ of PPPN was determined to be
$5 \times 10^{5}$ and 2.5 by gel permeation chromatography combined with low-angle light scattering photometry. We did not measure $\bar{M}_{w}$ of PCPN and PFPN. However, we expect that they also have the values of $\bar{M}_{w}$ and $\bar{M}_{w} / \bar{M}_{n}$ similar to PPPN since the PPPN and PFPN samples were prepared from the same PCPN.

Films of PPPN and PFPN for dielectric measurement were prepared by compression molding at 400 and $340 \mathrm{~K}$, respectively. The crystallinity of the films thus prepared was measured as follows.

For the PPPN film, a density method was employed. The density of the film and that of an almost perfectly amorphous PPPN containing $5 \%$ unsubstituted chlorine were measured by a density gradient method at $25^{\circ} \mathrm{C}$ using aqueous solutions of zinc chloride as gradient forming liquids. The latter being determined to be $1.237 \mathrm{~g} \mathrm{~cm}^{-3}$ was assumed to be the density of $100 \%$ amorphous PPPN. The crystalline density was estimated from the X-ray crystallographic data given by Stroh. ${ }^{11}$

The crystallinity of PFPN was determined from the heat of the mesophase transition using the heat of transition reported by Schneider et al. ${ }^{7}$

The crystallinity of PCPN was determined from the jump of the heat capacity $\Delta C_{\mathrm{p}}$ at the glass transition temperature with a differential scanning calorimeter (DSC) (Rigaku Denki model 8055 and 8058). We determined the value of $\Delta C_{p}$ for a perfectly amorphous PCPN from $\Delta C_{\mathrm{p}}$ measurement of a specimen after directly immersing it into liquid nitrogen.

The glass transition temperatures of the films were also determined with DSC. The results of the characterization were summarized in Table I.

Dielectric measurement was carried out with a capacitance bridge (General Radio 1615-A) and an automatic bridge (Yokogawa-HewlettPackard 4270A). Films of PPPN and PFPN for dielectric measurement were coated with evapolated gold to achieve tight contact between the films and the electrodes. The diam- 
Table I. Density $\rho$, crystallinity $\chi$, glass transition temperature $T_{\mathrm{g}}$, mesophase transition temperature $T_{1}$ and melting temperature $T_{\mathrm{m}}$ of the sample films

\begin{tabular}{|c|c|c|c|c|c|}
\hline \multirow{2}{*}{ Sample } & $\rho$ & $\chi$ & $T_{\mathrm{g}}$ & $T_{1}$ & $T_{\mathrm{m}}$ \\
\hline & $\mathrm{g} \mathrm{cm}^{-3}$ & $\%$ & K & $\mathrm{K}$ & $\mathrm{K}$ \\
\hline PCPN & 1.855 & 66 & 211 & - & 298 \\
\hline $\mathrm{PPPN}^{\mathrm{a}}$ & 1.351 & 61 & 263 & 400 & 620 \\
\hline PFPN & 1.475 & 78 & 233 & 347 & 513 \\
\hline
\end{tabular}

a $\bar{M}_{w}=5 \times 10^{5} ; \bar{M}_{w} / \bar{M}_{n}=2.5$ by LALS-GPC.

eter and thickness of the films were $50 \mathrm{~mm}$ and $0.5 \mathrm{~mm}$, respectively. Since the PCPN sample was soft, it was cast from a benzene solution in a cell type electrode, and then sandwitched with another electrode. The distance between the electrodes was controlled with a glass spacer.

\section{RESULTS AND DISCUSSION}

Dielectric Relaxations in PCPN, PPPN, and PFPN

Temperature dependences of the dielectric constant $\varepsilon^{\prime}$ and loss factor $\varepsilon^{\prime \prime}$ for PCPN, PPPN, and PFPN are shown in Figures $1-3$, respectively.

For PCPN, we see a loss maximum in the temperature range from 220 to $260 \mathrm{~K}$. Since the glass transition temperature of this polymer was observed around $211 \mathrm{~K}$ by DSC, we assigned this relaxation to the primary $\alpha$ process (glass transition process) due to the motion of Type-B dipoles. As seen in Figure 1, no secondary $(\beta)$ process was found in the range below $200 \mathrm{~K}$. PCPN exhibited high direct current (d.c.) conductivity in the temperature range above $250 \mathrm{~K}$. This is probably due to impurity ions such as $\mathrm{Cl}^{-}$produced by degradation of the polymer or the monomer. The increase in $\varepsilon^{\prime}$ of PCPN in the temperature range above $270 \mathrm{~K}$ is partly due to the fusion of the crystalline part and partly due to interfacial polarization of the sample around the

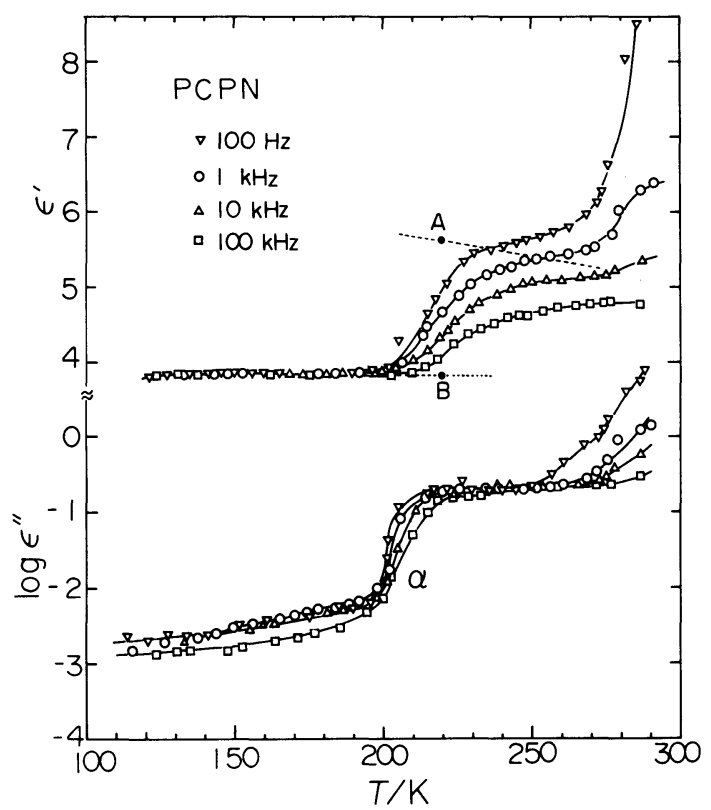

Figure 1. Temperature dependence of the dielectric constant $\varepsilon^{\prime}$ and loss $\varepsilon^{\prime \prime}$ for poly(dichloro phosphazene).

electrodes.

As shown in Figure 2, the ionic conductivity in PPPN was lower than that in PCPN. Thus, we could extend temperature range of measurement up to $460 \mathrm{~K}$ for this polymer. Around $280 \mathrm{~K}$, we observed a clear loss maximum. We named this process the $\alpha$ process. As in the case of PCPN, we assigned this loss maximum to the relaxation of type- $B$ dipoles in the amorphous region of the sample, since the $T_{\mathrm{g}}$ of PPPN was observed around $263 \mathrm{~K}$ by DSC. In the low temperature region below $200 \mathrm{~K}$, we see another small loss maximum. This secondary relaxation process was termed as the $\beta$ process. It is seen that at the mesophase transition point $T_{1}(=400 \mathrm{~K})$, both $\varepsilon^{\prime}$ and $\varepsilon^{\prime \prime}$ of PPPN increased suddenly. This indicates that the PPPN chains in the mesophase become more mobile than in the low temperature phase as discussed in the later section.

As seen in Figure 3, the $\varepsilon^{\prime}$ and $\varepsilon^{\prime \prime}$ curves for PFPN are similar to those for PPPN. Since the $T_{\mathrm{g}}$ of PFPN was $233 \mathrm{~K}$, we assigned the loss 


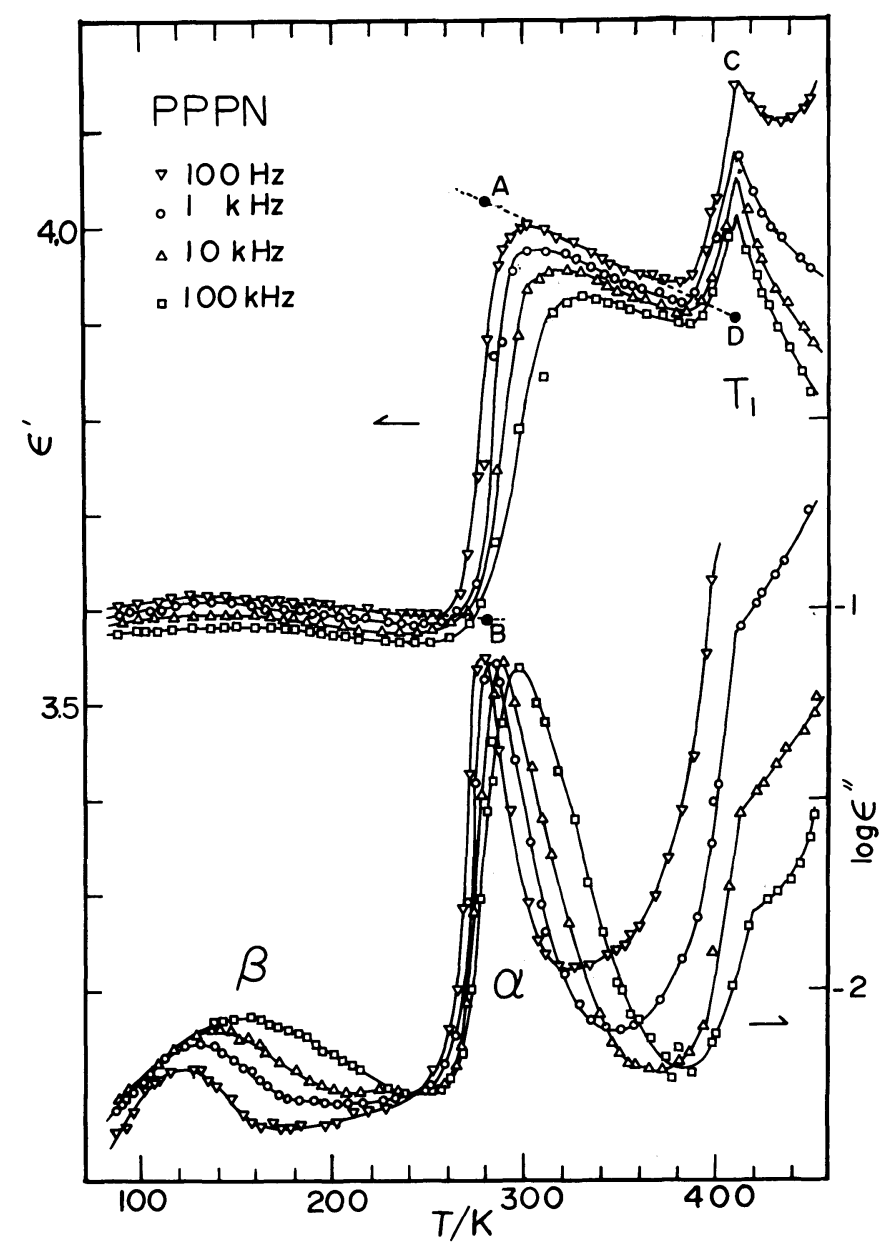

Figure 2. Temperature dependence of $\varepsilon^{\prime}$ and $\varepsilon^{\prime \prime}$ for poly(diphenoxy phosphazene).

maximum around $230 \mathrm{~K}$ to the primary $\alpha$ process and the loss around $120 \mathrm{~K}$ to the $\beta$ process. A sudden increase in $\varepsilon^{\prime}$ and $\varepsilon^{\prime \prime}$ is also seen at $T_{1}(=347 \mathrm{~K})$.

Generally, three molecular mechanisms for the secondary process are known: the local vibrational motion of the type $B$ dipole, the rotation of the side group, and the motion of the main chains or the side groups in the defects of the crystalline part. Since the $\beta$ process was seen only in PPPN and PFPN but not in PCPN which does not possess the type$\mathrm{C}$ dipole, we may assign the $\beta$ process to rotation of the side groups.
Plots of the loss maximum frequency $f_{\mathrm{m}}$ against the inverse of temperature for PCPN, PPPN, and PFPN are shown in Figure 4. It is known that the relaxation time for the primary process conforms to Vogel-Tamman equation. ${ }^{1}$ We also see this trend for the $\alpha$ processes of these samples. However, in our frequency range from 0.1 to $100 \mathrm{kHz}$, the Arrhenius plots are almost linear as shown in Figure 4. Therefore, we determined the activation energy for the $\alpha$ process assuming the plots are linear. The results are listed in Table II. The Arrhenius plots for the $\beta$ process for PPPN and PFPN also deviated from the linear re- 


\section{Dielectric Relaxation of Polyphosphazene}

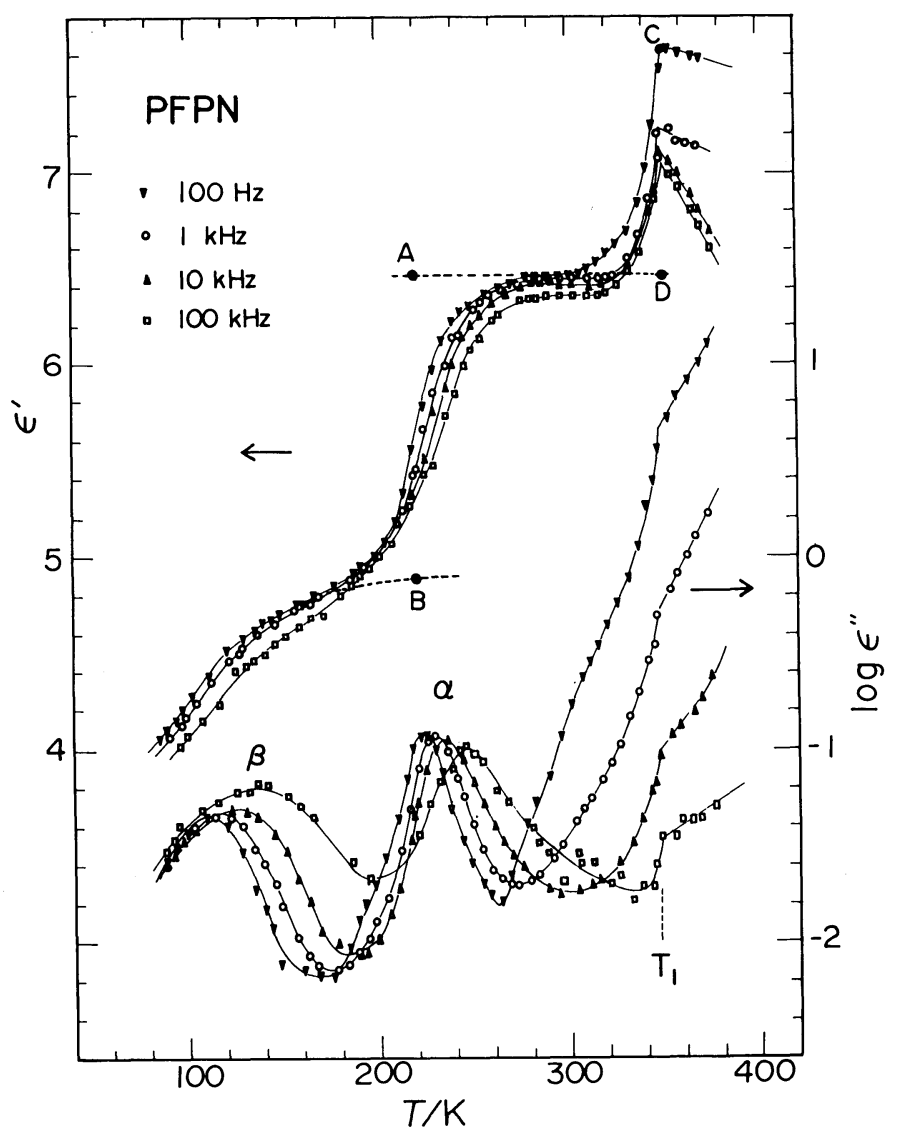

Figure 3. Temperature dependence of $\varepsilon^{\prime}$ and $\varepsilon^{\prime \prime}$ for poly(difluoroethoxy phosphazene).

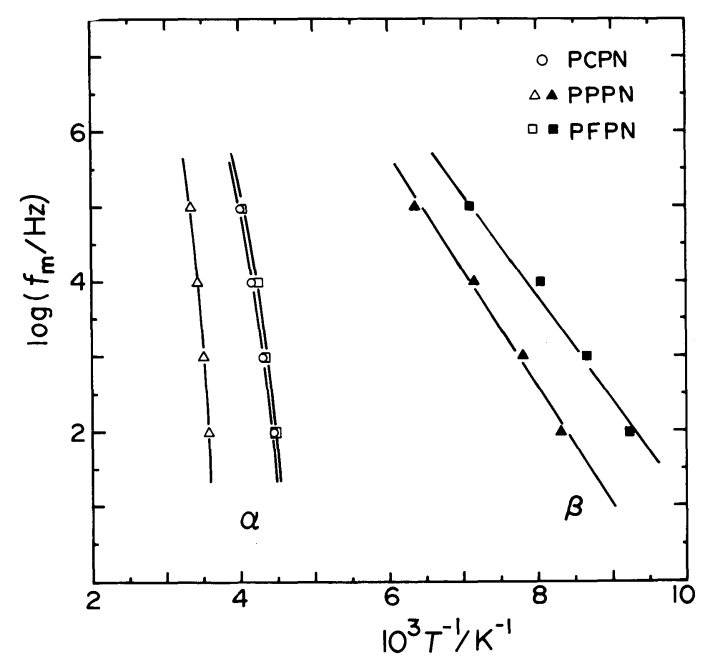

Figure 4. Transition map for PCPN, PPPN, and PFPN. The open and closed symbols represent the $\alpha$ and $\beta$ processes, respectively. lation. We determined the activation energy by drawing straight lines as shown in Figure 4. The results are also shown in Table II.

We found that PPPN and PFPN exhibit both the type $B$ and $C$ relaxations. As reported previously, ${ }^{3}$ PPPN exhibited the type-A relaxation in dilute solutions. Thus, we conclude that polyorganophosphazene having the type $\mathrm{C}$ dipole is classified as a type- $\mathrm{ABC}$ polymer.

\section{The $\alpha$ Process}

The relaxation strengths $\Delta \varepsilon_{\alpha}$ for the $\alpha$ process of PCPN, PPPN, and PFPN were determined from the jump of the $\varepsilon^{\prime}$ curve. The dotted lines in Figures $1-3$ indicate the extrapolation of the $\varepsilon^{\prime}$ curves above and below the $\alpha$ relaxation region. We determined the values of $\Delta \varepsilon_{\alpha}$ from 
Table II. Dielectric properties of polyphosphazene

\begin{tabular}{|c|c|c|c|c|c|c|c|}
\hline \multirow{3}{*}{ Sample } & $10^{-2} T \Delta \varepsilon_{\alpha}$ & $\mu_{\mathrm{B}}$ & $E_{\mathrm{B}}$ & $\mu_{\mathrm{C}}$ & $E_{\mathrm{a}}$ & $U$ & $\phi$ \\
\hline & K & $\mathrm{D}$ & $\mathrm{kJ} \mathrm{mol}^{-1}$ & D & $\mathrm{kJ} \mathrm{mol}^{-1}$ & $\mathrm{~kJ} \mathrm{~mol}^{-1}$ & deg \\
\hline & \multicolumn{3}{|c|}{$\alpha$ process } & \multicolumn{4}{|c|}{$\beta$ process } \\
\hline PCPN & $4.0^{\mathrm{a}}$ & 0.98 & 100 & - & - & - & - \\
\hline PPPN & 1.23 & 0.90 & 140 & $0.40^{\mathrm{b}}$ & 29.9 & 2.8 & 33 \\
\hline PFPN & 3.5 & 1.59 & 160 & $0.59^{\mathrm{c}, \mathrm{d}}$ & 25.9 & 3.1 & 38 \\
\hline \multicolumn{8}{|c|}{$\begin{array}{l}\text { The experimental uncertainty is } \pm 1.2 \text {. } \\
\text { b at } 127 \mathrm{~K} \text {. } \\
\text { c at } 142 \mathrm{~K} \text {. } \\
\text { d Debye } \mathrm{Cm}=3.333 \times 10^{-30}\end{array}$} \\
\hline
\end{tabular}

the difference between the points $\mathrm{A}$ and $\mathrm{B}$ in these figures. For PCPN, this method gave an ambiguous value since the tail of the relaxation process existing in high temperatures overlaps with the $\alpha$ process. The error in determination of $\Delta \varepsilon$ for PCPN was estimated to be $c a .30 \%$. Since $\Delta \varepsilon_{\alpha}$ is approximately proportional to $T^{-1}$, the values of $T \Delta \varepsilon$ are listed in Table II. We apply the Onsager theory to calculate the effective dipole moment $\mu$ per monomer unit: ${ }^{12}$

$$
\Delta \varepsilon=\frac{4 \pi N \mu^{2} \varepsilon_{0}\left(\varepsilon_{\infty}+2\right)^{2}}{9 k_{\mathrm{B}} T\left(2 \varepsilon_{0}+\varepsilon_{\infty}\right)}
$$

where $N$ is the number of the dipoles in unit volume of the amorphous part; $\varepsilon_{0}$, the relaxed dielectric constant; $\varepsilon_{\infty}$, the unrelaxed dielectric constant; and $k_{\mathrm{B}} T$, the thermal energy. Assuming that the $\mathrm{B}$ dipoles in the amorphous region are effective for the $\alpha$ relaxation, we calculated $N$ with $\chi$ given in Table I. The effective dipole moments $\mu_{\mathrm{B}}$ for the $\alpha$ process thus determined are listed in Table II.

The molecular structure of polyphosphazene with $\mathrm{Cl}$ and $\mathrm{O}-\mathrm{R}$ group is shown in Figure 5, where $\mathrm{R}$ represents phenyl and alkyl groups. The perpendicular dipole moment $\mu_{+}$ of the monomer unit of PCPN should be equal to the vector sum of the bond moments $\mu$ for the $\mathrm{P}-\mathrm{Cl}, \mathrm{P}=\mathrm{N}$, and $\mathrm{P}-\mathrm{N}$ bonds. The $\mathrm{P}-\mathrm{Cl}$ bond moment $\mu(\mathrm{P}-\mathrm{Cl})$ was reported to be

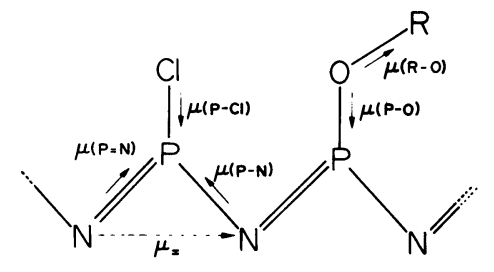

Figure 5. Analysis of the dipole moment of polyphosphazene.

$0.8 \mathrm{D},{ }^{13}$ but the values of the $\mathrm{P}=\mathrm{N}$ and $\mathrm{P}-\mathrm{N}$ bond moments $(\mu(\mathbf{P}-\mathbf{N}))$ are not reported. The parallel component $\mu_{=}$of the dipole moment per monomer unit is also given by the vector sum as shown in Figure 5. It is noted that the Cl-P (or $\mathrm{P}-\mathrm{O}$ ) bond is out of the $\mathrm{N}-\mathrm{P}-\mathrm{N}$ plane. If we assume that the $P$ atom is positively charged since electro-negativity of $\mathrm{N}$ is higher than $\mathrm{P}, \mu_{=}$and $\mu_{+}$may be given by

$$
\begin{aligned}
\mu_{=}= & {[\mu(\mathrm{P}=\mathrm{N})-\mu(\mathrm{P}-\mathrm{N})] \sin (\theta / 2) } \\
\mu_{+}= & {[\mu(\mathrm{P}=\mathrm{N})-\mu(\mathrm{P}-\mathrm{N})] \cos (\theta / 2) } \\
& -2 \mu(\mathrm{P}-\mathrm{Cl}) \cdot \cos (\psi / 2)
\end{aligned}
$$

where $\theta$ and $\psi$ denote the $\mathbf{N}=\mathbf{P}-\mathrm{N}$ and $\mathrm{Cl}-\mathrm{P}-\mathrm{Cl}$ angles, and were reported to be $115^{\circ}$ and $99^{\circ}$, respectively, by Chatani and Yatsuyanagi. ${ }^{14}$ Previously we determined $\mu_{=}$ to be $6.7 \mathrm{D}^{3}$ Using these values of $\mu_{=}$and $\mu(\mathrm{P}-\mathrm{Cl})$, we estimated $\mu_{+}$to be $3.2 \mathrm{D}$.

It is known that the segmental motion is associated with a co-operative motion of a unit 

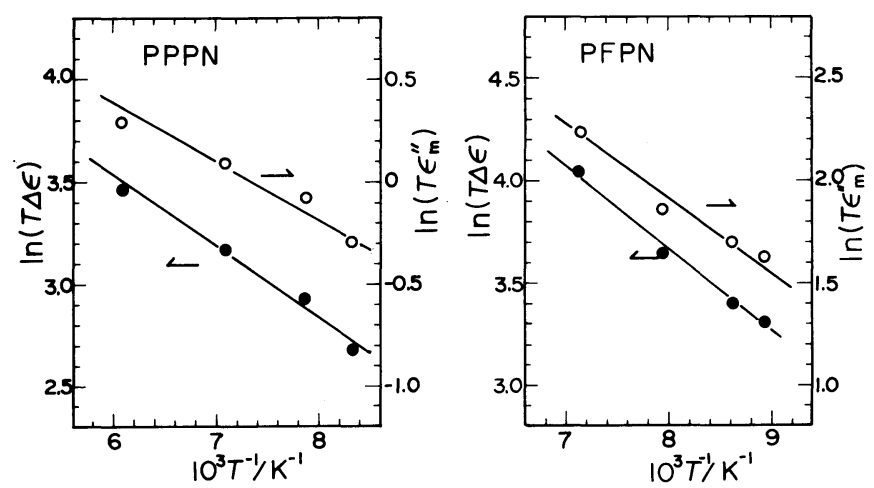

Figure 6. Temperature dependence of the relaxation strength $\Delta \varepsilon_{\mathrm{C}}$ and maximum value $\varepsilon_{\mathrm{m}}^{\prime \prime}$ of the loss factor for the $\beta$ processes of PFPN and PPPN.

composed of several numbers of the perpendicular dipoles. Thus, it is not surprising even if the observed $\mu_{\mathrm{B}}$ does not coincide with $\mu_{+}$. According to the Kirkwood theory, ${ }^{15} \mu_{\mathrm{B}}^{2}$ is equal to $g \mu_{+}^{2}$, where $g$ is the factor representing an orientational correlation among the dipole moments in the co-operative motional unit. From the above results, $g$ becomes 0.10 indicating a stronger trend toward antiparallel orientation of the type-B dipoles than ordinary organic polymers whose $g$ is $c a .0 .4 .^{16}$

For the analysis of PPPN and PFPN, we need the value of the $\mathrm{P}-\mathrm{O}$ bond moment $\mu(\mathrm{P}-\mathrm{O})$, which, however, has not been reported. Therefore, we estimated the perpendicular dipole moment to be 2.85 and $5.02 \mathrm{D}$ for PPPN and PFPN, respectively, assuming that the $g$ factor for PPPN and PFPN is 0.10 .

\section{The $\beta$ Relaxation}

The relaxation strength $\Delta \varepsilon_{\mathrm{C}}$ for the $\beta$ process was determined from the area of the $\varepsilon^{\prime \prime} v s .1 / T$ curve using the following relation:

$$
\Delta \varepsilon_{\mathrm{C}}=\left(2 E_{\mathrm{a}} / \pi R\right) \int_{0}^{\infty} \varepsilon^{\prime \prime} \mathrm{d}(1 / T) .
$$

We assumed that the $\Delta \varepsilon_{\mathrm{C}}$ thus determined corresponds to the value at the loss maximum temperature $T_{\max }$. The results are shown in Figure 6. From this data the effective dipole moment $\mu_{\mathrm{C}}$ for the $\beta$ process was calculated
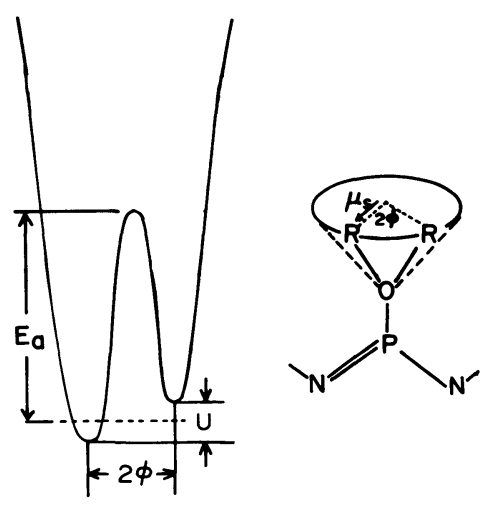

Figure 7. Two-site model for the rotation of the side group of polyorganophosphazene.

with eq 1 .

In order to estimate the strength of restriction for motion of the side group, we employ a two-site model ${ }^{1,17}$ as illustrated in Figure 7. Since we have no knowledge on the number of the sites in the real systems, the energy difference $U$ and the amplitude $\phi$ of the oscillation of the side group thus estimated give only rough measure of the restriction. More detailed information will be obtained by calculation of the potential energy as a function of the angle for the internal rotation. ${ }^{18,19}$

The energy barrier $E_{\mathrm{a}}$ in the model is determined from the activation energy for the $\beta$ process and given in Table II. The effective dipole moment $\mu_{\mathrm{C}}$ is given by 


$$
\begin{aligned}
& \mu_{\mathrm{C}}^{2}=4 K\left(\mu_{\mathrm{s}} \sin \phi\right)^{2} /(1+K)^{2} \\
& K=\exp \left(-U / k_{\mathrm{B}} T\right)
\end{aligned}
$$

where $\mu_{\mathrm{s}}$ is the dipole moment of the side group projected on the plane perpendicular to the rotational axis (see Figure 7).

First we determined $U$. The increase of $T \Delta \varepsilon_{\mathrm{C}}$ with increasing temperature reflects the increase of $K$. If $U$ is large compared with $k_{\mathrm{B}} T$, eq 1,5 , and 6 predict that logarithm of $T \Delta \varepsilon_{\mathrm{C}}$ is proportional to $-U / k_{\mathrm{B}} T$. This relation was tested in Figure 6 for PPPN and PFPN. In the determination of $\Delta \varepsilon$ from the area of the loss curve, there were ambiguities since the loss curve for the $\beta$ process was very broad and partly overlapped with the loss due to the $\alpha$ process. Thus, we also attemped to determine $U$ assuming that the maximum value $\varepsilon_{\mathrm{m}}^{\prime \prime}$ of the loss is proportional to the $\Delta \varepsilon_{\mathrm{C}}$. The plots of $\ln$ $T \varepsilon_{\mathrm{m}}^{\prime \prime} v s .1 / T$ is also shown in Figure 6. We see that the $U$ values determined from the two plots are almost similar. The average of the values of $U$ determined by the two methods is given in Table II.

The group moment for phenyl- $\mathrm{OCH}_{3}$ is reported to be $1.28 \mathrm{D}^{20}$ Thus, we estimated the phenyl-OP bonds of PPPN to be $1.3 \mathrm{D}$. Using this value and the C-O-P angle of $126^{\circ}$, $\mu_{\mathrm{s}}$ for PPPN was estimated to be $1.1 \mathrm{D}$. On the other hand, estimation of $\mu_{\mathrm{s}}$ for PFPN is more difficult than for PPPN since the side group has two degrees of freedom: the rotation around $\mathrm{P}-\mathrm{O}$ axis and that around $\mathrm{CF}_{3} \mathrm{CH}_{2}-\mathrm{O}$ axis. If the rotation around $\mathrm{P}-\mathrm{O}$ bond is assumed, $\mu_{\mathrm{s}}$ becomes $1.1 \mathrm{D}$ since the moment for alkyl-O bond is reported to be $1.28 \mathrm{D} .{ }^{20}$ If rotation of $\mathrm{CF}_{3}$ group is dominant, $\mu_{\mathrm{s}}$ becomes 2.2 D from the reported $\mathrm{CF}_{3}-\mathrm{CH}_{2}$ bond moment of $2.32 \mathrm{D}^{20}$ Using these values of $U$ and $\mu_{\mathrm{s}}$ estimated above, we calculated $\phi$ with eq 5 and 6. For PFPN, $\mu_{\mathrm{s}}=1.1$ and 2.2 led $\sin \phi>1$ and $\phi=38^{\circ}$, respectively. Thus, we adopted the latter value.

\section{Dielectric Behavior in the Mesophase}

The jump of $\varepsilon^{\prime}$ at $T_{1}$, i.e., the difference of $\varepsilon^{\prime}$ for the points $C$ and $D$ in Figures 2 and 3 may be ascribed to the two mechanisms: One is the onset of a new relaxation process named as the $\alpha^{\prime}$ process and the other is that the immobilized dipoles in the crystalline phase become mobile by the mesophase transition.

Above $T_{1}, \varepsilon^{\prime}$ of PPPN at $100 \mathrm{~Hz}$ increased with increasing temperature. This indicates that there exists a relaxation process in high temperature region. As reported previously, ${ }^{3}$ the normal mode process due to the type-A dipole of PPPN was observed in dilute solutions at $303 \mathrm{~K}$. We expect that in the bulk state, the relaxation time for the type-A dipole becomes much longer than in solution due to entanglement and the increase in the friction coefficient. Hence the relaxation of the type-A dipole is expected to occur at very high temperature. We may ascribe the $\alpha^{\prime}$ process to the normal mode process.

The jump of $\varepsilon^{\prime}$ at $T_{1}$ for the $\varepsilon^{\prime}$ curve at $100 \mathrm{kHz}$ may be due to the release of the dipole in the crystalline part, since the contribution of the type-A dipole is still very small at high frequency. If we assume that the content of the amorphous part does not change by the transition, then the increase in $\varepsilon^{\prime}$ should be ascribed to the orientational polarization in the liquid-crystalline part. Thus, the jump of $\varepsilon^{\prime}$ at $T_{1}$ at $100 \mathrm{kHz}$ may be given by

$$
\varepsilon^{\prime}\left(\text { above } T_{1}\right)-\varepsilon^{\prime}\left(\text { below } T_{1}\right)=\chi \Delta \varepsilon_{\text {meso }}
$$

where $\Delta \varepsilon_{\text {meso }}$ is the total relaxation strength in the perfectly pure mesophase.

We estimated $\Delta \varepsilon_{\text {meso }}$ in PFPN and PPPN to be 11 and 2.9 , respectively, assuming that the types $\mathrm{B}$ and $\mathrm{C}$ dipoles can move freely in the mesophase. The value of $\chi \Delta \varepsilon_{\text {meso }}$ thus calculated by eq 8 is much larger than the observed jump of the $\varepsilon^{\prime}$ which is equal to 0.7 and 0.13 for PFPN and PPPN, respectively. Accordingly the above result indicates that the motion of the dipoles in the mesophase is still restricted strongly. It seems to be reasonable to 
consider that in the mesophase the type-B dipole does not contribute to polarization since the chain has a extended structure in which the type-B dipoles cancel each other. If we assume that only the type- $\mathrm{C}$ dipole rotate freely, the theoretical value is still higher than the observed jump of $\varepsilon^{\prime}$. Thus, we conclude that the type-C dipoles in the mesophase rotate under a constraint.

\section{CONCLUSION}

(1) Poly(organophosphazene) exhibits the type-B and type-C relaxations in the solid state. Since it also exhibits the type-A relaxation in dilute solution, this polymer is classified as a type $\mathrm{ABC}$ polymer.

(2) The $\beta$ relaxation in PPPN and PFPN is due to the relaxation of the type $\mathrm{C}$ dipoles on the side group.

(3) In the mesophase of PPPN and PFPN, the motion of the side group and the type- $B$ dipoles are restricted.

Acknowledgement. This work was supported in part by Grants-in-Aid for Scientific Research (C6055062) from the Ministry of Education, Science, and Culture of Japan. A support from the Institute of Polymer Research, Osaka University is also gratefully acknowledged.

\section{REFERENCES AND NOTES}

1. N. G. McCrum, B. E. Read, and G. Williams, "Anelastic and Dielectric Effects in Polymeric Solids," Wiley, New York, N. Y., 1967.

2. W. H. Stockmayer, Pure Appl. Chem., 15, 539 (1967).

3. S. Uzaki, K. Adachi, and T. Kotaka, Macromolecules, in press.

4. B. H. Zimm, J. Chem. Phys., 24, 269 (1956).

5. H. R. Allcock and R. L. Kugel, J. Am. Chem. Soc., 87, 4216 (1965).

6. H. R. Allcock, Chem. Rev., 72, 315 (1972).

7. N. S. Schneider, C. R. Desper, and J. J. Beres, "Liquid Crystalline Order in Polymers," A. Blumstein, Ed., Academic Press, New York, N. Y., 1978, Chapter 9.

8. G. Allen, C. J. Lewis, and S. M. Todd, Polymer, 11, 44 (1970).

9. R. E. Singler, N. S. Schneider, and G. L. Hagnauer, Polym. Eng. Sci., 15, 321 (1975).

10. S. M. Aharoni, J. Macromol. Sci.-Phys., B21, 105 (1982).

11. E. G. Stroh, Jr., Ph. D. Thesis, Pennsylvania State Univ., see also ref 7.

12. L. Onsager, J. Am. Chem. Soc., 58, 1486 (1936).

13. E. Bergmann and L. Engel, Z. Phys. Chem., B31, 232 (1931).

14. Y. Chatani and K. Yatsuyanagi, Macromolecules, 20, 1042 (1987).

15. J. G. Kirkwood, J. Chem. Phys., 7, 911 (1939).

16. see for example, S. B. Dev, A. M. North, and J. C. Reid, "Dielectric Properties of Polymers," F. E. Karasz, Ed., Plenum Press, New York, N. Y., 1972, p 217.

17. see for example, M. Kakizaki, H. Nakayama, and T. Hideshima, Polym. J., 18, 141 (1986).

18. N. Sanno, I. Murakami, and H. Yamamura, Polym. J., 8, 231 (1976).

19. A. Tanaka and Y. Ishida, J. Polym. Sci. B, 12, 335 (1974).

20. V. I. Minkin, O. A. Osipov, and Yu. A. Zhdanov, "Dipole Moments in Organic Chemistry," translated by B. J. Hazzard and W. E. Vaughan, Plenum Press, New York, N. Y., 1970. 